\title{
EFECTOS DE ENZIMAS E INOCULANTES SOBRE LA COMPOSICIÓN DEL ENSILAJE DE MAÍZ
}

\section{ENZYME AND INOCULANT EFFECTS ON CORN SILAGE COMPOSITION}

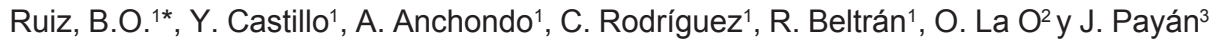

${ }^{1}$ Facultad de Zootecnia. Universidad Autónoma de Chihuahua. Investigación. Periférico Francisco R. Almada, Km. 1. CP 31031 Chihuahua. Chihuahua. México. *Correspondencia: oscaruiz@uach.mx ${ }^{2}$ Instituto de Ciencia Animal. Departamento de Ciencias Biofisiológicas. San José de las Lajas. La Habana. Cuba.

${ }^{3}$ Instituto Nacional de Investigaciones Forestales, Agrícolas y Pecuarias. Campo Experimental Delicias. Carretera Delicias-Rosales km 2. Delicias. Chihuahua. México.

\section{Palabras clave adicionales}

Aditivos. Digestibilidad in vitro.

\section{RESUMEN}

El tratamiento de ensilajes con enzimas fibrolíticas e inoculantes bacterianos ha mostrado aumentar digestibilidad, fermentación y estabilidad aeróbica de varios forrajes. El objetivo fue evaluar el efecto en la composición química y características de fermentación en el ensilaje con los híbridos DK641® (DK641), Eagle 238W® (238W), Golden Harvest EX313 $($ EX313), Golden Harvest H9403 ${ }^{\circledR}$ (H9403), Pioneer $32 \mathrm{R} 25 \AA$ (32R25), Pioneer $31 \mathrm{G} 98 \AA$ (31G98) y Producers $725 \AA$ (725) tratados con tres aditivos. Los híbridos se sembraron en parcelas experimentales de 1 ha en Chihuahua, México. La cosecha se realizó en la etapa de madurez con $1 / 3$ de llenado del grano tomándose muestras representativas para la elaboración de microsilos $(1,3 \pm 0,1 \mathrm{~kg})$ por $42 \mathrm{~d}$. Se evaluaron cuatro tratamientos de microsilos: el control (CT) con $20 \mathrm{ml}$ de agua destilada y tres tratamientos con aditivo; SA con $0,0065 \mathrm{~g}$ de Sill All $4 \times 4 \otimes$ (L. plantarum, P. acidilactii, E. faecium y $B$. salivarus), BS con $0,182 \mathrm{~g}$ de Bio-sile ${ }^{\circledR}(L$. plantarum y $P$. pentosaceus) y FB con $0,31 \mathrm{~g}$ de Fibrozyme ${ }^{\circledR}$ (xylanases y $T$. viride). Se determinó materia seca (MS), proteína bruta (PB), fibra detergente neutro (FDN), fibra detergente ácido (FDA), celulosa (CE), hemicelulosa (HEM), digestibilidad in vitro de la materia seca (DIVMS) digestibilidad in vitro de la materia orgánica (DIVMO), $\mathrm{pH}$ y ácido láctico (AL). La inoculación (SA y BS) incrementó los contenidos de FDN en los

Recibido: 16-2-07. Aceptado: 1-10-07.

\section{AdDitional KeYWORDS \\ Additives. In vitro digestibility.}

ensilajes de los materiales 31G98 y DK641 $(p<0,05)$. Asimismo, la adición de $S A$ incrementó el contenido de PB en H9403, 238W y EX313 $(p<0,05)$. La adición de FB disminuyó el contenido de FDN en 31G98, H9403 y 238W; pero disminuyó el pH en DK641 y EX313 e incrementó la concentración de AL en 32R25 $(p<0,05)$. Los resultados mostraron un efecto positivo al tratar el ensilaje con aditivos; no obstante, este varía dependiendo de las características propias de cada uno de los híbridos utilizados.

\section{SUMMARY}

Treating silages with fibrolytic enzymes and bacterial inoculants has been shown to improve digestibility, fermentation and aerobic stability of a variety of forages. The objective of this experiment was to evaluate the effects of three additives on chemical composition and fermentation characteristics of silage from seven different hybrids: DK641 ${ }^{\mathrm{TM}}$, Eagle 238W ${ }^{\mathrm{T} \mathrm{M}}$, Golden Harvest EX313 ${ }^{\mathrm{TM}}$, Golden Harvest H9403 ${ }^{\mathrm{TM}}$, Pioneer $32 \mathrm{R} 25^{\mathrm{TM}}$, Pioneer $31 \mathrm{G}^{\mathrm{TM}}$, and Producers $725^{\mathrm{TM}}$. Corn plants were planted in experimental plots (1 ha each) in Chihuahua, Mexico and harvested at stage of maturity of $1 / 3$ of milkline. Whole corn plants were cut and subsamples were chopped and ensiled in mini silos $(1.3 \pm 0.1 \mathrm{~kg})$ for $42 \mathrm{~d}$. Treatments were control: CT: $20 \mathrm{ml}$ distilled water; SA: $0.0065 \mathrm{~g}$ Sill 
All ${ }^{\mathrm{TM}} 4 \times 4$ (L. plantarum, P. acidilactii, E. faecium, and $B$. salivarus); BS: $0.182 \mathrm{~g}$ Bio-sile ${ }^{\mathrm{TM}}(L$. plantarum and $P$. pentosaceus) and FB: $0.31 \mathrm{~g}$ Fibrozyme $^{\mathrm{TM}}$ (xylanases and $T$. viride). Silage samples were analyzed for dry matter (DM), crude protein (CP), neutral detergent fiber (NDF), acid detergent fiber (ADF), cellulose (CE), hemicellulose $(\mathrm{HE})$, in vitro dry matter digestibility (IVDMD), in vitro organic matter digestibility (IVOMD), $\mathrm{pH}$ and lactic acid (LA). Inoculation with SA and BS increased content of NDF of silage of hybrids $31 \mathrm{G} 98$ and DK641 $(p<0.05)$. Addition of SA increased CP of silages from H9403, 238W and EX313 $(p<0.05)$. However, FB decreased NDF concentration of $31 \mathrm{G} 98, \mathrm{H} 9403$ and 238W, lowered $\mathrm{pH}$ of DK641 and EX313 and increased concentration of LA content of EX313 and increased concentration of LA content of EX313 $(p<0.05)$. No effect on DM and OM digestibility was observed for FB, but when BS and SA were added a significant difference $(p<0.05)$ was observed for the hybrids DK641, 32R25 and Producers 725. These show that when applied at ensiling certain inoculants and fibrolytic enzymes can improve various chemical characteristics of corn silage, however, effects vary depending of the particular corn hybrid.

\section{INTRODUCCIÓN}

El ensilaje de maíz es uno de los forrajes conservados más importantes y versátiles en el mundo. Es una mezcla única de grano y fibra digestible, que constituye una de las principales fuentes energéticas para la alimentación de rumiantes. El ensilaje es un método de preservación del forraje húmedo basado en convertir carbohidratos solubles en ácidos orgánicos, principalmente ácido láctico, bajo condiciones anaeróbicas por medio de la acción de bacterias (Filya, 2003). De acuerdo con De la Raza-Delgado (2005) la calidad fermentativa de un ensilado depende de la naturaleza del forraje original y en el desarrollo de la técnica empleada, además del clima, la estación, el estado de madurez, la composición química y botánica, etc. Sin embargo, existen otros aspectos inherentes al propio forraje que limitan su aptitud para ser ensilado o ensilabilidad (Muck y Bolsen, 1991; Muck, 2004). Jones
(1995) reportó que para la optimización de este proceso es recomendable la utilización de ciertos tipos de aditivos, buscando mejorar el valor nutritivo del maíz. Dentro de los aditivos destacan los inoculantes bacterianos, los cuales contienen bacterias productoras de ácido láctico que se agregan a la población bacterial natural para ayudar a garantizar una fermentación rápida y eficiente en el silo (Muck y Kung Jr, 1997).

Las enzimas son otro tipo de aditivos las cuales contienen una gran variedad de proteínas que pueden actuar degradando los carbohidratos estructurales de las plantas, así como el almidón que se encuentra en los granos y, como consecuencia, se mejora la fermentación y la utilización por el animal (Muck y Bolsen, 1991). De acuerdo con Nadeau et al. (2000a) el efecto más consistente de las enzimas degradadoras de pared celular, como las celulasas y hemicelulasas, es que reducen la concentración de los carbohidratos estructurales. Esta baja concentración de pared celular esta asociada con mayores consumos de materia seca y por ende en una mejoría del comportamiento animal. Al respecto, Hristov y McAllister (2002) notaron que al agregar aditivos se presentaba una mejor preservación del forraje ensilado, una alta concentración de azúcares solubles y una mayor degradabilidad ruminal. Además, otros autores como Dean et al. (2005) mostraron que la aplicación de enzimas fibrolíticas podían aumentar la digestibilidad, fermentación y estabilidad aeróbica del ensilaje del pasto bermuda.

Los inoculantes han tenido un gran auge en los últimos años en diversos países; sin embargo, en muchas regiones del mundo, se carece de información comparativa y detallada al respecto que especifique los efectos que sobre el ensilaje de maíz se producen al utilizar un determinado aditivo. Además, se requiere información sobre la conveniencia de su utilización considerando la gran variedad de híbridos de maíz que se utilizan en México para ensilaje. El objetivo de este trabajo fue evaluar el efecto de dos inocu- 


\section{ADITIVOS MEJORADORES DEL ENSILAJE DE MAÍZ}

lantes microbianos y un aditivo enzimático sobre la fermentación y valor nutritivo del ensilaje de siete híbridos comerciales de maíz. Los resultados permitirán ofrecer nuevas herramientas a los productores de ensilaje de maíz para que seleccionen aquel aditivo que les ofrezca una mayor relación beneficio-costo como consecuencia de elevar la calidad nutritiva del forraje ensilado.

\section{MATERIAL Y MÉTODOS}

Los híbridos fueron sembrados en el rancho La Casita, municipio de Bachíniva, Chihuahua, México. El rancho se encuentra entre los $28^{\circ} 97^{\prime}$ latitud norte y $107^{\circ} 73^{\prime}$ longitud oeste, a una altitud de $2020 \mathrm{msnm}$. La temperatura media anual es de $13,6^{\circ} \mathrm{C}$ con máximas de $34,3^{\circ} \mathrm{C}$ y mínimas de $-10,6^{\circ} \mathrm{C}$ y una precipitación media anual de $449 \mathrm{~mm}$. Se utilizaron siete híbridos de maíz; DK641® (DK641), Eagle 238W® (238W), Golden Harvest EX313® (EX313), Golden Harvest H9403® (H9403), Pioneer 32R25® (32R25), Pioneer $31 \mathrm{G} 98 \AA(31 \mathrm{G} 98)$ y Producers $725 \AA$ (725). La siembra se realizó en siete parcelas de una hectárea, utilizando un total de 20 surcos por híbrido. El suelo presenta un $\mathrm{pH}$ de 6,9, una textura franco-arcillosa y sin problemas de sales $\left(<0,1 \mathrm{Sm}^{-1}\right)$ y de sodio ( $<12,0$ de relación de adsorción de sodio). La preparación del suelo, la siembra, los riegos y la fertilización se realizó de acuerdo a lo sugerido por el Campo Experimental Sierra de Chihuahua del Instituto Nacional de Investigaciones Forestales, Agrícolas y Pecuarias. A cada híbrido se le realizó un corte en la etapa de madurez de $1 / 3$ avance de la línea de leche, cosechando diez plantas de maíz de un surco y repitiendo la operación a intervalos de cinco surcos. Se obtuvieron cuatro repeticiones de cada uno de los híbridos. Luego se transportaron al laboratorio de investigación en nutrición animal perteneciente a la Facultad de Zootecnia donde se mantuvieron en refrigeración a $4^{\circ} \mathrm{C}$ durante 12 horas. Con las muestras de forraje se elaboraron microsilos. El material se picó a un tamaño de partícula de $2,54 \mathrm{~cm}$ con una picadora de forraje John Deere (Modelo 3975). El forraje una vez picado y homogenizado $(1,3 \pm 0,1 \mathrm{~kg}$, base húmeda) se colocó en bolsas de plástico transparente selladas al vacío, para luego ser almacenado por 42 días dentro de bolsas de plástico de color negro para evitar su exposición a la luz solar. Transcurrido este período los microsilos se abrieron y fueron congelados a $-20^{\circ} \mathrm{C}$ para preservar sus características y realizar los análisis correspondientes.

Se evaluaron cuatro tratamientos: el control (CT) donde únicamente se adicionó $20 \mathrm{ml}$ de agua destilada, un tratamiento con la adición de $0,0065 \mathrm{~g}$ de Sill All 4x4TM (Lactobacillus plantarum, 12,6×109 UFC/g, Pediococus acidilacti 4,2×109 $\mathrm{UFC} / \mathrm{g}$, Enterococus faecium $2,1 \times 10^{9} \mathrm{UFC} / \mathrm{g}, \mathrm{Baci}$ llus salivarus $2,1 \times 10^{9} \mathrm{de} \mathrm{UFC} / \mathrm{g}$ y $\beta$-glucanasas; Alltech, U.S.A) (SA), un tratamiento con la adición de $0,182 \mathrm{~g}$ de Bio-sile ${ }^{\mathrm{TM}}(L$. plantarum y $P$. pentosaceus, 9x $10^{9} \mathrm{UFC} / \mathrm{g}$; Hansen, U.S.A ) (BS) y un cuarto tratamiento con la adición de $0,31 \mathrm{~g}$ de Fibrozyme ${ }^{\mathrm{TM}}$ (xylanases, $100 \mathrm{XU} / \mathrm{g}$ provenientes de extractos solubles de la fermentación de Aspergillus niger y Trichoderma viride; Alltech, EE.UU.) (FB). La aplicación de los tratamientos se realizó con un atomizador manual, disolviendo los productos en $20 \mathrm{ml}$ de agua destilada y asperjándolos de manera uniforme sobre el forraje. Previo a la elaboración de los microsilos se determinó el contenido de MS en el forraje, colocando $20 \mathrm{~g}$ del material que se muestreó antes de almacenar en cada microsilo, en una estufa de aire forzado a $100^{\circ} \mathrm{C}$ por 24 horas. $\mathrm{Al}$ material ensilado se le realizaron determinaciones de MS, así como de $\mathrm{pH}$ (Tejada, 1992) y ácido láctico (Taylor, 1996). Una muestra del ensilaje (300 g) se secó en una estufa a $55^{\circ} \mathrm{C}$ por 72 horas, se molió a un tamaño de partícula de $1,0 \mathrm{~mm}$ en un molino Wiley y se analizó para PB (AOAC, 1990), FDN, FDA, HEM y CEL (Goering y Van Soest, 1970) así como DIVMS y DIVMO de 
acuerdo a la metodología de dos fases propuesta por Tilley y Terry (1963). Todas las determinaciones se hicieron por triplicado. Para el análisis de la información se utilizó un diseño completamente al azar realizando un análisis de varianza (ANOVA) para todas las variables mediante el procedimiento GLM del SAS (SAS, 2002). La comparación de medias se realizó aplicando la prueba de Tukey usando un nivel de significación de 0,05 . El modelo utilizado para la evaluación de los híbridos fue:

$$
\mathrm{Y}_{\mathrm{ij}}=\mu+\mathrm{V}_{\mathrm{i}}+\mathrm{e}_{\mathrm{ij}}
$$

donde:

$\mathrm{Y}_{\mathrm{ij}}=$ variable respuesta,

$\mu=$ media general

$V_{i}=$ efecto del i-ésimo híbrido,

$\mathrm{e}_{\mathrm{ij}}=$ error experimental.

\section{RESULTADOSYDISCUSIÓN}

Para mejorar el proceso de ensilaje, evitando el deterioro aeróbico, varios tipos de aditivos han sido desarrollados. Estos son productos muy ventajosos porque son fáciles de usar, seguros y sobre todo que no contaminan el ambiente. Los resultados sobre el efecto de los dos inoculantes bacterianos o bacterias lácticas (BAL) y el aditivo enzimático en las características bromatológicas y de fermentación de los híbridos de maíz se presentan en las tablas I a VII. La inoculación con BAL (SA y BS), incrementó significativamente $(\mathrm{p}<0,05)$ los contenidos de FDN en relación al control, en el ensilaje de maíz 31 G98 (tabla I); FDN y FDA en DK 641 (tabla II); mientras que en H9403 (tabla III) también se observó un incremento $(p<0,05)$ en FDN y FDA, pero sólo en el caso de BS. En el resto de los híbridos no existió efecto de la inoculación sobre FDN y FDA. Resultados encontrados por Kung et al. (1993) al evaluar ensilaje de maíz tratados con dos inoculantes (Ecosyl ${ }^{\circledR}$ y Pioneer ${ }^{\circledR}$ 1174) muestran que la PB, FDA, FDN y contenidos minerales fueron similares para todos los ensilajes, sin embargo la FDN del control fue mayor $(44,1 \%)$ que el ensilaje tratado con Ecosyl ${ }^{\circledR}$ y con Pioneer ${ }^{\circledR} 1174$ $(43,1$ y $43,8 \%)$, no así en FDA donde los ensilajes inoculados tuvieron mayores porcentajes que el control (24,0 y 24,5 vs. $22,7 \%)$. La mayor concentración de FDN cuando se inocula con SA y BS, concuerda con lo reportado por Rodrígues et al. (2002) quie-

Tabla I. Efectos de dos inoculantes bacterianos y un aditivo enzimático sobre el ensilaje del híbrido de maíz Pioneer 31 G98. (Effects of two bacterial inoculants and a enzymatic additive on hybrid corn silage Pioneer 31G98).

\begin{tabular}{lccccc}
\hline & Control (CT) & Sill Al (SA) & Biosile (BS) & Fibrozyme (FB) & E.E. \\
\hline MS (\%) & 19,5 & 20,2 & 19,5 & 20,1 & $\pm 0,70$ \\
MO (\%) & 94,3 & 93,7 & 93,6 & 93,5 & $\pm 0,30$ \\
PB (\%) & 906 & 9,01 & 900 & 9,02 & $\pm 0,04$ \\
FDN (\%) & $67,6^{\mathrm{b}}$ & $71,1^{\mathrm{a}}$ & $69,7^{\mathrm{a}}$ & $64,1^{\mathrm{c}}$ & $\pm 0,43$ \\
FDA (\%) & $43,4^{\mathrm{ab}}$ & $44,8^{\mathrm{a}}$ & $44,7^{\mathrm{ab}}$ & $42,8^{\mathrm{b}}$ & $\pm 0,40$ \\
Celulosa (\%) & 26,7 & 27,2 & 26,3 & 28,0 & $\pm 0,49$ \\
Hemicelulosa (\%) & $24,2^{\mathrm{b}}$ & $26,2^{\mathrm{a}}$ & $25,0^{\mathrm{ab}}$ & $21,3^{\mathrm{c}}$ & $\pm 0,37$ \\
DIVMS (\%) & 63,5 & 63,7 & 63,4 & 66,8 & $\pm 1,04$ \\
DIVMO (\%) & 63,7 & 64,6 & 64,1 & 65,5 & $\pm 1,27$ \\
Ph & 3,53 & 3,50 & 3,50 & 3,50 & $\pm 0,02$ \\
Ácido láctico (\%) & 11,2 & 11,5 & 11,0 & 10,5 & $\pm 0,96$ \\
\hline
\end{tabular}

abcMedias en la misma fila con distinto superíndice son diferentes $(p<0,05)$. 


\section{ADITIVOS MEJORADORES DELENSILAJE DE MAÍZ}

Tabla II. Efectos de dos inoculantes bacterianos y un aditivo enzimático sobre el ensilaje del híbrido de maíz Dekalb DK64. (Effects of two bacterial inoculants and a enzymatic additive on hybrid corn silage Dekalb DK64).

\begin{tabular}{lccccc}
\hline & Control (CT) & Sill Al (SA) & Biosile (BS) & Fibrozyme (FB) & E.E. \\
\hline MS (\%) & 20,2 & 21,4 & 22,4 & 19,7 & $\pm 0,77$ \\
MO (\%) & $94,1^{\mathrm{a}}$ & $93,1^{\mathrm{b}}$ & $93,5^{\mathrm{ab}}$ & $93,6^{\mathrm{a}}$ & $\pm 0,16$ \\
PB (\%) & 9,2 & 9,2 & 9,1 & 9,1 & $\pm 0,05$ \\
FDN (\%) & $64,4^{\mathrm{b}}$ & $69,0^{\mathrm{a}}$ & $67,6^{\mathrm{a}}$ & $63,0^{\mathrm{b}}$ & $\pm 0,63$ \\
FDA (\%) & $38,6^{\mathrm{b}}$ & $43,9^{\mathrm{a}}$ & $42,1^{\mathrm{a}}$ & $38,5^{\mathrm{b}}$ & $\pm 0,45$ \\
Celulosa (\%) & $24,5^{\mathrm{ab}}$ & $22,9^{\mathrm{bc}}$ & $22,3^{\mathrm{c}}$ & $25,9^{\mathrm{a}}$ & $\pm 0,41$ \\
Hemicelulosa(\%) & 25,8 & 25,1 & 25,4 & 24,5 & $\pm 0,32$ \\
DIVMS (\%) & $64,8^{\mathrm{b}}$ & $65,1^{\mathrm{b}}$ & $69,7^{\mathrm{a}}$ & $69,5^{\mathrm{a}}$ & $\pm 0,81$ \\
DIVMO (\%) & 65,6 & 65,2 & 69,4 & 68,4 & $\pm 1,19$ \\
pH & $3,63^{\mathrm{a}}$ & $3,60^{\mathrm{ab}}$ & $3,60^{\mathrm{ab}}$ & $3,55^{\mathrm{b}}$ & $\pm 0,02$ \\
Ácido láctico(\%) & $9,7^{\mathrm{b}}$ & $10,9^{\mathrm{ab}}$ & $9,7^{\mathrm{b}}$ & $12,1^{\mathrm{a}}$ & $\pm 0,48$ \\
\hline
\end{tabular}

abcMedias en la misma fila con distinto superíndice son diferentes $(p<0,05)$.

nes evaluaron el efecto de la inoculación de BAL en ensilaje de sorgo y encontraron un incremento en la FDN del ensilaje que fue tratado con Sill All®. Ranjit et al. (2002) mencionan que el ensilaje de maíz tratado con un inoculante comercial (Biotal $\left.{ }^{\circledR}\right)$ presentó mayores contenidos de FDN y FDA, en relación a un ensilaje no inoculado y atribuyeron dicho efecto a una mayor utili- zación, por parte de las BAL, de las fracciones más solubles de la planta de maíz en relación a las fracciones fibrosas. Respecto a la DIVMS, no se encontró diferencia $(\mathrm{p}>0,05)$ de SA y BS respecto al tratamiento control en los híbridos 31G98, H9403,238W y EX313; por el contrario, en los híbridos DK-641, 32R 25 y Producers 725 (tabla VI) se observó diferencia significativa $(\mathrm{p}<0,05)$

Tabla III. Efectos de dos inoculantes bacterianos y un aditivo enzimático sobre el ensilaje del híbrido de maíz Golden Harvest H9403. (Effects of two bacterial inoculants and a enzymatic additive on hybrid corn silage Golden Harvest H9403).

\begin{tabular}{|c|c|c|c|c|c|}
\hline & Control (CT) & Sill Al (SA) & Biosile (BS) & Fibrozyme (FB) & E.E. \\
\hline MS (\%) & $19,8^{a}$ & $19,1^{\mathrm{ab}}$ & $18,1^{b}$ & $18,9^{\mathrm{ab}}$ & $\pm 0,36$ \\
\hline $\mathrm{MO}(\%)$ & 92,5 & 91,9 & 92,2 & 92,3 & $\pm 0,24$ \\
\hline PB (\%) & $9,5^{\mathrm{b}}$ & $9,8^{a}$ & $9,5^{b}$ & $9,5^{\mathrm{b}}$ & $\pm 0,04$ \\
\hline FDN (\%) & $65,4^{b}$ & $66,2^{\mathrm{ab}}$ & $67,7^{a}$ & $62,9^{c}$ & $\pm 0,34$ \\
\hline FDA (\%) & $41,6^{b}$ & $41,2^{b}$ & $43,3^{a}$ & $42,4^{\mathrm{ab}}$ & $\pm 0,34$ \\
\hline Celulosa (\%) & $26,6^{b}$ & $25,2^{b}$ & $26,0^{b}$ & $30,0^{\mathrm{a}}$ & $\pm 0,48$ \\
\hline Hemicelulosa (\%) & $23,8^{a}$ & $25,0^{a}$ & $24,4^{a}$ & $20,5^{b}$ & $\pm 0,44$ \\
\hline DIVMS (\%) & 60,4 & 60,3 & 63,3 & 64,1 & $\pm 0,99$ \\
\hline DIVMO (\%) & 61,3 & 60,5 & 62,4 & 62,7 & $\pm 0,87$ \\
\hline $\mathrm{pH}$ & $3,56^{b}$ & $3,60^{\mathrm{ab}}$ & $3,56^{b}$ & $3,70^{a}$ & $\pm 0,02$ \\
\hline Ácido láctico (\%) & 10,7 & 12,5 & 13,0 & 13,1 & $\pm 0,56$ \\
\hline
\end{tabular}

abcMedias en la misma fila con distinto superíndice son diferentes $(p<0,05)$. 
RUIZ, CASTILLO, ANCHONDO, RODRÍGUEZ, BELTRÁN, LA OY PAYÁN

para BS, siendo en este último híbrido donde se obtuvo el mayor porcentaje de digestibilidad $(73,5)$, aunque no mostró diferencia respecto al control. Contrario a lo anterior, Filya (2003) trabajando con ensilajes de sorgo no encontró respuesta en digestibilidad de materia orgánica y FDN a inoculaciones con BAL, sin dar explicación de la ausencia de efecto. Por otra lado, FB incrementó el porcentaje de DIVMS comparado con el tratamiento control en la totalidad de los híbridos, aunque sólo en el caso de Dekalb se presentó diferencia significativa $\mathrm{p}=0,05$ ), resultados que están en desacuerdo al reporte de Nadeau et al. (2000b) quienes al inocular celulasas disminuyeron la digestibilidad de FDN por $18 \%$. Sin embargo, ensilajes de pasto Bermuda tratados con enzimas fibrolíticas exógenas (Dean et al., 2005), promovieron una mayor digestibilidad de la DIVMS en comparación con otras enzimas, atribuyendo este efecto a la acción sinergística de celulasas y hemicelulasas. La adición de FB disminuyó el contenido de FDN ( $\mathrm{p}<0,05)$, con respecto al control, en los ensilajes de 31G98 (tabla I), H9403 (tabla III) y $238 \mathrm{~W}$ (tabla IV); mientras que en el resto de los híbridos no existió efecto algu- no. La disminución de FDN probablemente se debió a la acción de hemicelulasas, como lo sugiere su menor contenido de HEM, sin embargo estas disminuciones en el contenido de FDN no se ven reflejadas significativamente $(\mathrm{p} \geq 0,05)$ en la DIVMS de los ensilajes tratados, llegando a la misma conclusión de Nadeau et al. (2000b). Lo anterior también concuerda con lo reportado por Sheperd y Kung (1996) quienes evaluaron un producto enzimático (Cornzyme ${ }^{\circledR}$ ) a base de celulasas y hemicelulasas en el ensilaje de maíz, encontrando una disminución en FDN y FDA. Spolaestra et al.(1992), Nadeau et al. (2000a) y Dean et al. (2005) también reportaron una disminución en FDN después de la adición de enzimas celulolíticas a ensilajes de maíz, alfalfa y pasto, mencionando estos últimos que ciertas enzimas fibrolíticas pueden aumentar la digestibilidad, fermentación y estabilidad aeróbica del ensilaje de pasto bermuda. Por el contrario, Chen et al. (1994) al evaluar ensilaje de maíz inoculado con una enzima experimental no encontraron diferencias en FDA, FDN y celulosa.

En relación al contenido de $\mathrm{PB}$, se observó un incremento en el contenido respecto

Tabla IV. Efectos de dos inoculantes bacterianos y un aditivo enzimático sobre el ensilaje del hibrido de maiz Eagle $238 \mathrm{~W}$. (Effects of two bacterial inoculants and a enzymatic additive on hybrid corn silage Eagle 238W).

\begin{tabular}{lccccc}
\hline & Control (CT) & Sill Al (SA) & Biosile (BS) & Fibrozyme (FB) & E.E. \\
\hline MS (\%) & 22,7 & 23,4 & 22,2 & 23,2 & $\pm 0,47$ \\
MO (\%) & $93,8^{\text {ab }}$ & $93,0^{\mathrm{b}}$ & $94,0^{\mathrm{a}}$ & $93,4^{\mathrm{ab}}$ & $\pm 0,20$ \\
PB (\%) & $8,1^{\mathrm{b}}$ & $8,6^{\mathrm{a}}$ & $8,7^{\mathrm{a}}$ & $8,1^{\mathrm{b}}$ & $\pm 0,05$ \\
FDN (\%) & $68,0^{\mathrm{a}}$ & $68,0^{\mathrm{a}}$ & $66,9^{\mathrm{ab}}$ & $64,8^{\mathrm{b}}$ & $\pm 0,51$ \\
FDA (\%) & $42,3^{\mathrm{ab}}$ & $42,1^{\mathrm{ab}}$ & $40,1^{\mathrm{b}}$ & $42,7^{\mathrm{a}}$ & $\pm 0,52$ \\
Celulosa (\%) & $29,8^{\mathrm{a}}$ & $25,2^{\mathrm{b}}$ & $22,0^{\mathrm{a}}$ & $29,34^{\mathrm{a}}$ & $\pm 0,49$ \\
Hemicelulosa (\%) & $25,8^{\mathrm{a}}$ & $25,9^{\mathrm{a}}$ & $26,8^{\mathrm{a}}$ & $22,2^{\mathrm{b}}$ & $\pm 0,40$ \\
DIVMS (\%) & 64,5 & 61,4 & 67,0 & 65,6 & $\pm 1,28$ \\
DIVMO (\%) & 65,0 & 61,8 & 66,9 & 64,4 & $\pm 1,58$ \\
pH & 3,56 & 3,65 & 3,60 & 3,63 & $\pm 0,02$ \\
Ácido láctico (\%) & 9,4 & 8,7 & 8,5 & 10,2 & $\pm 0,54$ \\
\hline
\end{tabular}

abcMedias en la misma fila con distinto superíndice son diferentes $(p<0,05)$.

Archivos de zootecnia vol. 58, núm. 222, p. 168. 


\section{ADITIVOS MEJORADORES DELENSILAJE DE MAÍZ}

Tabla $V$. Efectos de dos inoculantes bacterianos y un aditivo enzimático sobre el ensilaje del híbrido de maíz Pioneer 32R25. (Effects of two bacterial inoculants and a enzymatic additive on hybrid corn silage Pioneer 32R25).

\begin{tabular}{lccccc}
\hline & Control (CT) & Sill Al (SA) & Biosile (BS) & Fibrozyme (FB) & E.E. \\
\hline MS (\%) & 23,7 & 21,9 & 24,3 & 22,5 & $\pm 0,56$ \\
MO (\%) & $94,5^{\mathrm{a}}$ & $93,5^{\mathrm{b}}$ & $93,8^{\mathrm{ab}}$ & $93,2^{\mathrm{b}}$ & $\pm 0,15$ \\
PB (\%) & 7,6 & 7,9 & 7,6 & 7,9 & $\pm 0,09$ \\
FDN (\%) & 62,9 & 62,5 & 60,6 & 63,4 & $\pm 0,71$ \\
FDA (\%) & $37,2^{\mathrm{b}}$ & $40,1^{\mathrm{a}}$ & $37,4^{\mathrm{b}}$ & $40,4^{\mathrm{a}}$ & $\pm 0,57$ \\
Celulosa (\%) & $21,5^{\mathrm{c}}$ & $20,4^{\mathrm{c}}$ & $24,5^{\mathrm{b}}$ & $27,0^{\mathrm{a}}$ & $\pm 0,40$ \\
Hemicelulosa (\%) & $25,7^{\mathrm{a}}$ & $22,4^{\mathrm{b}}$ & $23,2^{\mathrm{b}}$ & $23,0^{\mathrm{b}}$ & $\pm 0,41$ \\
DIVMS (\%) & $64,3^{\mathrm{bc}}$ & $63,5^{\mathrm{c}}$ & $68,2^{\mathrm{a}}$ & $67,4^{\mathrm{ab}}$ & $\pm 0,86$ \\
DIVMO (\%) & 65,3 & 64,3 & 67,6 & 66,2 & $\pm 0,90$ \\
pH & $3,53^{\mathrm{b}}$ & $3,66^{\mathrm{a}}$ & $3,60^{\mathrm{a}}$ & $3,66^{\mathrm{a}}$ & $\pm 0,02$ \\
Ácido láctico (\%) & $8,5^{\mathrm{b}}$ & $9,6^{\mathrm{ab}}$ & $9,5^{\mathrm{ab}}$ & $10,4^{\mathrm{a}}$ & $\pm 0,39$ \\
\hline
\end{tabular}

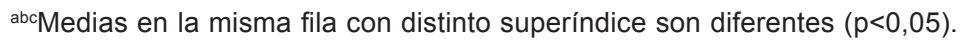

al control, en los ensilaje H9403 (tabla I) y 238W (tabla IV) con la adición de SA, mientras que la aplicación de BS hizo lo mismo en 238W ( $<<0,05)$. En el ensilaje de EX313 (tabla VII) se observó que los tres tratamientos presentaron contenidos mayores de $\mathrm{PB}$ con respecto al control $(\mathrm{p}<0,05)$. Meeske $e t$ al. (2002) evaluaron el efecto de la inocula- ción en el ensilaje de maíz y encontraron resultados similares a los del presente estudio, atribuyendo el mayor contenido de PB a una menor degradación de la proteína y menor pérdida de $\mathrm{N}$ derivado de a una fermentación más eficiente durante el proceso de ensilado. Sin embargo, resultados contradictorios (Chen et al., 1994) fueron en-

Tabla VI. Efectos de dos inoculantes bacterianos y un aditivo enzimático sobre el ensilaje del hibrido de maíz Producers 725. (Effects of two bacterial inoculants and a enzymatic additive on hybrid corn silage Producers 725).

\begin{tabular}{lccccr}
\hline & Control (CT) & Sill Al (SA) & Biosile (BS) & Fibrozyme (FB) & E.E. \\
\hline MS (\%) & 25,7 & 25,3 & 23,1 & 24,4 & $\pm 0,81$ \\
MO (\%) & $94,9^{\mathrm{a}}$ & $94,4^{\mathrm{a}}$ & $94,1^{\mathrm{b}}$ & $94,1^{\mathrm{b}}$ & $\pm 0,15$ \\
PB (\%) & $7,5^{\mathrm{b}}$ & 7,3 & 7,3 & 7,2 & $\pm 0,07$ \\
FDN (\%) & $60,6^{\mathrm{a}}$ & $57,9^{\mathrm{a}}$ & $54,3^{\mathrm{b}}$ & $60,6^{\mathrm{a}}$ & $\pm 0,71$ \\
FDA (\%) & $36,8^{\mathrm{ab}}$ & $34,2^{\mathrm{b}}$ & $34,2^{\mathrm{b}}$ & $37,6^{\mathrm{a}}$ & $\pm 0,64$ \\
Celulosa (\%) & $22,6^{\mathrm{b}}$ & $21,7^{\mathrm{b}}$ & $19,8^{\mathrm{c}}$ & $25,4^{\mathrm{a}}$ & $\pm 0,35$ \\
Hemicelulosa (\%) & $23,8^{\mathrm{a}}$ & $23,7^{\mathrm{a}}$ & $20,1^{\mathrm{b}}$ & $23,0^{\mathrm{a}}$ & $\pm 0,44$ \\
DIVMS (\%) & $69,4^{\mathrm{a}}$ & $63,5^{\mathrm{b}}$ & $73,5^{\mathrm{a}}$ & $73,0^{\mathrm{a}}$ & $\pm 1,12$ \\
DIVMO (\%) & $69,6^{\mathrm{a}}$ & $65,3^{\mathrm{b}}$ & $72,1^{\mathrm{a}}$ & $72,3^{\mathrm{a}}$ & $\pm 0,96$ \\
pH & 3,53 & 3,50 & 3,50 & 3,50 & $\pm 0,01$ \\
Ácido láctico (\%) & 9,6 & 8,7 & 9,9 & 10,2 & $\pm 0,50$ \\
\hline
\end{tabular}

abcMedias en la misma fila con distinto superíndice son diferentes $(p<0,05)$. 
RUIZ, CASTILLO, ANCHONDO, RODRÍGUEZ, BELTRÁN, LA OY PAYÁN

Tabla VII. Efecto de dos inoculantes bacterianos y un aditivo enzimático sobre el ensilaje del híbrido de maíz Golden Harvest EX313. (Effects of two bacterial inoculants and a enzymatic additive on hybrid corn silage Golden Harvest EX313).

\begin{tabular}{lccccc}
\hline & Control (CT) & Sill Al (SA) & Biosile (BS) & Fibrozyme (FB) & E.E. \\
\hline MS (\%) & 22,8 & 23,0 & 23,0 & 21,1 & $\pm 0,46$ \\
MO (\%) & $94,1^{\mathrm{a}}$ & $93,8^{\mathrm{ab}}$ & $93,2^{\mathrm{b}}$ & $94,3^{\mathrm{a}}$ & $\pm 0,18$ \\
PB (\%) & $7,23^{\mathrm{b}}$ & $8,14^{\mathrm{a}}$ & $8,17^{\mathrm{a}}$ & $8,24^{\mathrm{a}}$ & $\pm 0,03$ \\
FDN (\%) & $65,2^{\mathrm{a}}$ & $62,8^{\mathrm{ab}}$ & $60,1^{\mathrm{b}}$ & $62,4^{\mathrm{bc}}$ & $\pm 0,59$ \\
FDA (\%) & $41,5^{\mathrm{a}}$ & $39,2^{\mathrm{a}}$ & $37,5^{\mathrm{b}}$ & $39,9^{\mathrm{ab}}$ & $\pm 0,61$ \\
Celulosa (\%) & $22,2^{\mathrm{b}}$ & $21,1^{\mathrm{b}}$ & $21,9^{\mathrm{b}}$ & $27,9^{\mathrm{a}}$ & $\pm 0,37$ \\
Hemicelulosa (\%) & 23,7 & 23,6 & 22,7 & 22,6 & $\pm 0,33$ \\
DIVMS (\%) & 66,9 & 66,4 & 70,0 & 68,8 & $\pm 0,91$ \\
DIVMO (\%) & 67,9 & 66,6 & 69,4 & 68,7 & $\pm 1,04$ \\
pH & $3,70^{\mathrm{a}}$ & $3,70^{\mathrm{a}}$ & $3,70^{\mathrm{a}}$ & $3,50^{\mathrm{b}}$ & $\pm 0,00$ \\
Ácido láctico (\%) & 9,6 & 9,2 & 10,6 & 10,4 & $\pm 0,72$ \\
\hline
\end{tabular}

abcMedias en la misma fila con distinto superíndice son diferentes $(p<0,05)$.

contrados al tratar una mezcla de ensilaje de gramíneas y leguminosas con un complejo de enzima-inoculante. La enzima FB no mostró ningún efecto sobre $\mathrm{PB}$, a excepción de Golden Harvest $(\mathrm{p} \leq 0,05)$ en donde se registró un aumento de $12 \%$. Todos los valores de $\mathrm{pH}$ se mantuvieron dentro de los rangos normales para ensilajes de maíz (Kung et al., 1993; Filya, 2003; Kung y Shaver, 2001). Sin embargo, la inoculación tendió a disminuir el pH del ensilaje de los híbridos 31G98, DK641 y Producers 725 (tabla VI) sin encontrar diferencias $(p>0,05)$ a excepción deFB en DK641 p $\leq 0,05)$, en el híbrido EX313 el pH se mantuvo igual para SA, BS y tratamiento control pero no para FB donde disminuyó significativamente $(\mathrm{p} \leq 0,05)$. En el resto de los híbridos el $\mathrm{pH}$ fue mayor en los tratamientos con inoculantes (SA, BS y FB) lo cual indica que la fermentación bacteriana fue la adecuada mejorando la preservación del ensilaje. Al respecto, Kung y Shaver 2001, mencionan que raramente un ensilaje de maíz tiene un $\mathrm{pH}$ por arriba de 4,2; el cual puede ser asociado con ensilajes extremadamente se$\cos (>42 \%$ materia seca), e indican un rango de 3,7 a 4,2 como normal en un ensilaje de maíz con un $30-40 \%$ de materia seca.

Similares resultados a nuestro trabajo han sido reportados por Colombatto et al. (2003) quienes encontraron menores valores de $\mathrm{pH}$ en el ensilaje de maíz tratado con enzimas en relación a otro no tratado y señalan que dicho efecto se debe a una mayor disposición de sustrato para la fermentación, promovida por la acción de las enzimas adicionadas al momento de ensilar. Resultados contrarios fueron reportados por Bolsen et al. (1980), aplicando una enzima comercial a ensilaje de maíz, no encontraron cambios en el pH del ensilaje o producción de lactato comparado con el control, el ensilaje tratado tuvo menor estabilidad aeróbica y el tratamiento de enzima tendió a incrementar la relación de agua $\mathrm{N}$ soluble del total del $\mathrm{N}$, indicando incremento en la proteolisis. El uso de aditivos deben de producir grandes cantidades de ácido láctico en un periodo corto de tiempo con el fin de estabilizar el ensilaje y con pérdidas mínimas. En este trabajo, el ácido láctico tendió a aumentar en los híbridos H9403 y 32R25 sin mostrar significación estadística $(\mathrm{p} \leq 0,05)$ al tratarse con BAL; el resto de los híbridos mantuvo valores similares comparados con el control, excepto en el híbrido 238W donde disminuyeron (SA y BS) con respecto al control. Al aplicar FB al ensilaje, 


\section{ADITIVOS MEJORADORES DEL ENSILAJE DE MAÍZ}

se encontró mayor respuesta $(p<0,05)$ en los híbridos DK641 y 32R25. El efecto positivo de las inoculaciones con BAL y FB nos indica que este procedimiento acelera la tasa inicial de fermentación láctica, reduciendo el $\mathrm{pH}$ a valores mínimos para favorecer una baja degradación proteica y pérdidas fermentativas. Al respecto Chen et al. (1994), trabajando con ensilaje de maíz control y ensilaje de maíz tratado con una enzima experimental, encontraron una disminución en ácido láctico del tratamiento con la enzima experimental, esto debido probablemente a que el forraje de maíz original estaba relativamente seco (38\% MS). Por el contrario ensilaje de maíz tratado con Ecosyl ${ }^{\circledR}$ $(p>0,05)$ tuvo un mayor porcentaje de ácido láctico que el tratamiento control (Kung et al., 1993).

\section{CONCLUSIONES}

El valor nutritivo y la fermentación del ensilaje de maíz pueden ser mejorados por el tratamiento con inoculantes bacterianos y enzimas fibrolíticas. La adición de inoculantes bacterianos (BAL) incrementó los contenidos de FDN en los híbridos Pioneer 31 G98 (tabla V) y Dekalb DK641, mientras que cuando se inoculó con enzima (FB) este

\section{BIBLIOGRAFÍA}

AOAC. 1990. Official methods of analyses. $15^{\text {th }}$ edition. Association of Official Analytical Chemist. Arlington. Virginia.

Bolsen, K.K., H.J. Ilg and D.E. Axe. 1980. Additives for corn silage. J. Anim. Sci., 51(Suppl. 1): 230. (Abstr.).

Chen, J., M.R. Stokes and C.R. Wallace. 1994. Effects of enzime-inoculant system on preservation and nutritive value of hay crop and corn silages. J. Dairy Sci., 77: 501-512.

Colombatto, D., F.L. Mould, M.K. Bhat, R.H. Phipps and E. Owen. 2003. In vitro evaluation of fibrolytic enzymes as additives for maize (Zea mays L.) silage. II: Effects on rate of acidification, fibre degradation during ensiling and rumen fermentation. Anim. Feed Sci. Technol., 111: 129-143. contenido disminuyó en los híbridos Pioneer 31G98, Golden Harvest H9403 y Eagle $238 \mathrm{~W}$, pero disminuyendo los valores de $\mathrm{pH}$ en los híbridos Dekalb DK641 y Golden Harvest EX313. Asimismo, la adición enzimática incrementó la concentración de ácido láctico en Pioneer 32R25. A excepción de estos tres últimos casos, ninguno de los aditivos mejoró sustancialmente los parámetros de fermentación en el ensilaje de maíz en comparación con el testigo. En lo que se refiere a la proteína bruta la adición de Sill All ${ }^{\mathrm{TM}}$ mejoró su concentración en los híbridos Golden Harvest H9403, Eagle 238W y Golden Harvest EX313. El efecto de los aditivos usados fue muy heterogéneo entre los siete híbridos evaluados, lo que indica que la respuesta obtenida al utilizar este tipo de productos comerciales está influenciada por las característica propias de los forrajes utilizados, por lo que es recomendable seleccionar tanto al híbrido como al aditivo adecuado para lograr ventajas en la relación costo-beneficio. Finalmente y ante la escasa información acerca de la evaluación de ensilados tratados biológicamente después de su apertura, es recomendable incluir su estabilidad aeróbica como un indicativo de la efectividad de los diferentes aditivos ensayados

Dean, D.B., A.T. Adesogan, N. Krueger and R.C. Littell. 2005. Effect of fibrolytic enzymes on the fermentation characteristics, aerobic stability, and digestibility of bermudagrass silage. J. Dairy Sci., 88: 994-1003.

De la Raza-Delgado, B. 2005. El ensilado en zonas húmedas y sus indicadores de calidad. IV Jornadas de Alimentación Animal. Laboratorio de Mouriscade. Lalín (Pontevedra).

Filya, I. 2003. The effect of Lactobacillus buchneri and Lactobacillus plantarum on the fermentation, aerobic stability, and ruminal degradability of low dry matter corn and sorghum silages. J. Dairy Sci., 86: 3575-3581.

Goering, H.K. and P.J. Van Soest. 1970. Forage fiber analyses (apparatus, reagents, proce- 


\section{RUIZ, CASTILLO, ANCHONDO, RODRÍGUEZ, BELTRÁN, LA OY PAYÁN}

dures and some applications). USDA-ARS. Agricultural Handbook 379. U.S. Government Printing Office. Washington, D.C.

Hristov, A.N. and T.A. McAllister. 2002. Effect of inoculants on whole-crop barley silage fermentation and dry matter disappearance in situ. J. Anim. Sci., 80: 510-516.

Jones, R. 1995. Role of biological additives in crop conservation. In: Biotechnology in the feed industry. Proceedings of Altech's $11^{\text {th }}$ Annual Symposium. Ed. Lyons, T.P. y K.A. Jaques. Nottingham University Press. Nottingham, UK. p. 465-482.

Kung Jr., L., J.H. Chen, E.M. Kreck and K. Knutsen. 1993. Effect of microbial inoculants on the nutritive nalue of corn silage for lactating dairy cows. J. Dairy Sci., 76: 3763-3770.

Kung, L. and R. Shaver. 2001. Interpretation and use of silage fermentation. Analysis reports. In: focus on forage. Wisconsin Team Forage. Vol. 3: No 13.

Meeske, R., G.D. van der Merwe, J.F. Greyling and C.W. Cruywagen. 2002. The effect of the addition of a lactic acid bacterial inoculant to maize at ensiling on silage composition, silage intake, milk production and milk composition. S. Afr. J. Anim. Sci., 32: 263-270.

Muck., R.E. and L. Kung Jr. 1997. Effects of silage additives on ensiling. In: Proceedings Silage: Field to Feedbunk. Conference. Feb. 11-13, Hershey, Pennsylvania. p. 187-199.

Muck, R.E. and K.K. Bolsen. 1991. Silage preservation and silage additive products. In Field guide for hay and silage management in North America. K.K. Bolsen, J.E. Baylor y M.E. McCullough. Ed. Natl. Feed Ingred. Assoc. West Des Moines IA. p. 105.

Muck, R. 2004. Effects of corn silage inoculants on aerobic stability. Trans. ASAE, 47: 1011-1016.

Nadeau, E.M.G., D.R. Buxton, J.R. Russell, M.J.
Allison and J.W. Young. 2000a. Enzyme, bacterial inoculant, and formic acid effects on silage composition of orchardgrass and alfalfa. J. Dairy Sci., 83: 1487-1502.

Nadeau, E.M.G., J.S. Russell and D.R. Buxton. 2000b. Intake, digestibility, and composition of orchardgrass and alfalfa silages treated with cellulose, inoculant, and formic acid fed to lambs. J. Anim. Sci., 78: 2980-2989

Ranjit, N.K., C.C. Taylor and L. Kung Jr. 2002. Effect of Lactobacillus buchneri 40788 on the fermentation, aerobic stability and nutritive value of maize silage. Grass Forage Sci., 57: 73-81.

Rodrigues, M.P. H., A.L. Senatore, S.J. Tavares, J. Martins, C. de Sousa and F.R. de Lima. 2002. Effects of microbial inoculants on chemical composition and fermentation characteristics of sorghum silage. R. Bras. Zootec., 31: 23732379.

SAS Institute. 2002. SAS User's Guide. SAS Institute Inc, Cary, NC. USA.

Sheperd, M.G. and L. Kung Jr. 1996. An enzyme additive for corn silage: effects on silage composition and animal performance. J. Dairy Sci., 79: 1760-1766.

Spolaestra, S.F., P.G. van Wikselar and B. Harder. 1992. The effects of ensiling whole crop maize with a multi-enzyme preparation on the chemical composition of the resulting silages. J. Sci. Food Agric., 60: 223.

Taylor, K. 1996. A simple colorimetric assay for muramic acid and lactic acid. Appl. Biochem. Biotech., 56: 49-58.

Tejada, I. 1992. Control de calidad y análisis de alimentos para animales. Sistema de educación continua en producción animal A.C. México, D.F.

Tilley, J.M. and R.A. Terry. 1963. A two stage technique for the in vitro digestion of forage crops. J. Brit. Grass. Soc., 18: 104-111.

Archivos de zootecnia vol. 58, núm. 222, p. 172. 